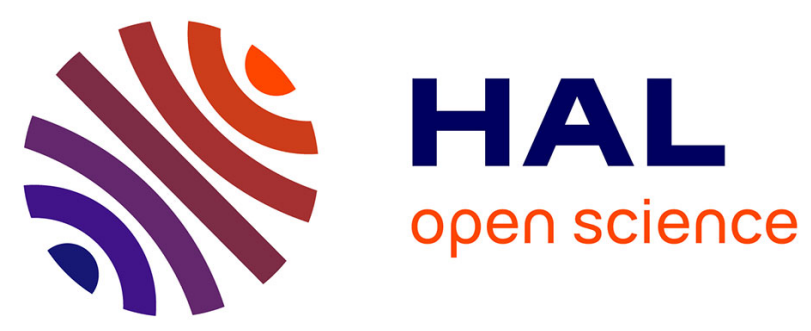

\title{
LIMITING TEMPERATURES OF EXCITED NUCLEI : STATIC AND DYNAMICAL ASPECTS
}

\author{
P. Bonche, D. Vautherin, M. Veneroni
}

\section{To cite this version:}

P. Bonche, D. Vautherin, M. Veneroni. LIMITING TEMPERATURES OF EXCITED NUCLEI : STATIC AND DYNAMICAL ASPECTS. International Conference on Heavy Ion Nuclear Collisions in the Fermi Energy Domain Hicofed 86, 1986, Caen, France. pp.C4-339-C4-350, 10.1051/jphyscol:1986437 . jpa-00225803

\section{HAL Id: jpa-00225803 https://hal.science/jpa-00225803}

Submitted on 1 Jan 1986

HAL is a multi-disciplinary open access archive for the deposit and dissemination of scientific research documents, whether they are published or not. The documents may come from teaching and research institutions in France or abroad, or from public or private research centers.
L'archive ouverte pluridisciplinaire HAL, est destinée au dépôt et à la diffusion de documents scientifiques de niveau recherche, publiés ou non, émanant des établissements d'enseignement et de recherche français ou étrangers, des laboratoires publics ou privés. 


\section{LIMITING TEMPERATURES OF EXCITED NUCLEI : STATIC AND DYNAMICAL ASPECTS}

P. BONCHE, D. VAUTHERIN* and M. VÉNÉRONI*

DPhG/SPhT, CEN-Saclay, F-91191 Gif-sur-Yvette Cedex, France

*Division de physique Théorique+, Institut de physique

Nucléaire, F-91406 Orsay Cedex, France

Résumé - Des calculs statiques et dynamiques de noyaux à haute température sont présentés et discutés dans le cadre de 1 'approximation de champ moyen.

Abstract - Some static and dynamical calculations of hot nuclei are discussed within the framework of the mean field approximation.

The phase diagram of nuclear matter is believed to exhibit the typical structure of a Van der Waals'fluid /1,2/. In particular this diagram shows a critical point, whose temperature $T_{C}$ is about $20 \mathrm{MeV}$ and below which the minimum value of the free energy corresponds to an equilibrium between a liquid and a vapor phase. Whether the corresponding liquid-vapor transition is observable in heavy-ion collisions has been the subject of much discussion. In contrast with the case of static infinite nuclear matter, a realistic description of highly excited nuclei, as they are produced in heavy-ion collisions, should include finite size, surface and Coulomb effects, as well as the dynamics of the collision. With the aim of exploring some of these effects, we intend in the following to compare the results of some static and dynamical calculations within the mean field approximation at finite temperature. This approximation is characterized by the replacement of the exact density operator by an uncorrelated one of the form

$$
D=\frac{1}{Z} \exp \left(-\sum_{i} \alpha_{i} a_{i}^{+} a_{i}\right)
$$

where $a_{j}^{+}$and $a_{j}$ are the nucleon creation and annihilation operators in the orbitals $\left|\varphi_{i}\right\rangle, Z$ being a normalization factor. We will therefore assume that the singleparticle degrees of freedom have been thermalized at some stage of the collision by the residuat interactions.

\section{I - STATIC MEAN FIELD CALCULATIONS OF HOT NUCLEI}

For Hartree-Fock calculations at finite temperature, the population of the individual states in the continuum, prevents the direct determination of $D$ by minimizing the free energy. A way to solve this problem is to perform mean field calculations for an equilibrium between a nucleus and an external vapor $/ 3 /$. (For an alternative approach see ref. $/ 4 /$ ). However this method makes it necessary to identify unambiguously the nucleus and vapor contributions.

i) Isolating the nucleus from its vapor.

It was shown in ref. /3/ that the Hartree-Fock equations at finite temperature, for given values of the chemical potential $\mu$ and volume $V$, have in general two solutions. One describes the nucleus-vapor system whereas the second describes the vapor only. The nucleus grand potential was defined in ref. $/ 3 /$ as the difference

\footnotetext{
tLaboratoire associe au CNRS
} 
between the grand potentials of these two solutions; this difference was shown to have a well defined limit when $V$ goes to infinity.

For independent nucleons in a potential $U(r)$ this subtraction procedure gives the following formula for the grand potential of the nucleus

$$
\begin{aligned}
\Omega & =-\sum_{n<0} \log \left(1+e^{\alpha-\beta E_{n}}\right) \\
& -\frac{k T}{\pi} \sum_{\ell}(2 \ell+1) \int_{0}^{\infty} d E \log \left(1+e^{\alpha-\beta E} \frac{d \delta_{\ell}(E)}{d E}\right)
\end{aligned}
$$

where $\beta=1 / k T$ is the inverse temperature and $\alpha / \beta=\mu$ the chemical potential, while $E_{n}$ and $\delta_{\ell}(E)$ denote respectively the energies of the bound states and the phase shifts for the potential $U(r)$. This formula agrees with the expression of the second order virial coefficient derived in 1937 by Bethe and Uhlenbeck /5/.

The subtraction procedure of ref. $/ 3 /$ also contains as a special case the formula of Tubbs and Koonin $/ 6 /$, which was derived in the semi-classical approximation for independent nucleons in a potential. $U(r)$. It gives the nucleus grand potential $\Omega$ as $\Omega_{1}-\Omega_{2}$ with

$$
\begin{aligned}
& \Omega_{1}=-\frac{k T}{3 \pi^{2}}{\frac{2 m k T}{\hbar^{2}}}^{3 / 2} \int d \vec{r} F_{3 / 2}(\alpha-\beta U(r)) \\
& \Omega_{2}=-\frac{k T}{3 \pi^{2}}{\frac{2 m k T}{\hbar^{2}}}^{3 / 2} \int d \vec{r} F_{3 / 2}(\alpha)
\end{aligned}
$$

where $F_{3 / 2}$ is the Fermi integral of order $3 / 2$. This formula may be shown to be the semi-classical limit of eq. (2) $/ 3 /$.

ii) Limiting temperature.

When applied within a Hartree-Fock framework the subtraction procedure (3) predicts the existence of a limiting temperature $T_{\ell}$ beyond which an equilibrium with an external vapor is no longer possible. The value of this limiting temperature has been found to be of the order of $10 \mathrm{MeV}:$ in the case of lead-208, $T_{l}=11 \mathrm{MeV}$ for the Skyrme III interaction while it is $8 \mathrm{MeV}$ for the SKM interaction /3/. Because of the coulomb effects, these values are appreciably smaller than the corresponding ones in (neutral) nuclear matter. Systematic investigations using the Thomas-Fermi approximation / $7 /$ and the hot liquid drop model / $/$ have shown that $T_{\ell}$ decreases from 1 ight to heavy nuclei (see fig. 1 ). This decrease is also due to the Coulomb effects.

It can be shown that the limiting temperature $T_{l}$ is not larger than the temperature $T_{0}$ for which the nucleus binding energy vanishes. Indeed when the energy of the nucleus is positive, it becomes possible to lower the free energy by a scale transformation on the orbitals, the occupation numbers (i.e. the entropy) being kept fixed. In practice, numerical calculations have shown that $\mathrm{T}_{\ell}$ is always slightly smaller than $\mathrm{T}_{0}$ (see Table 2 of ref. $/ 3 /$ ).

iii) Lifetimes of hot nuclei.

In static calculations the density of the vapor is directly related to the hot nucleus lifetime, a low density corresponding to a long lifetime $/ 3 /$. Values of the lifetime of lead-208, calculated from this relation are given in table 1 


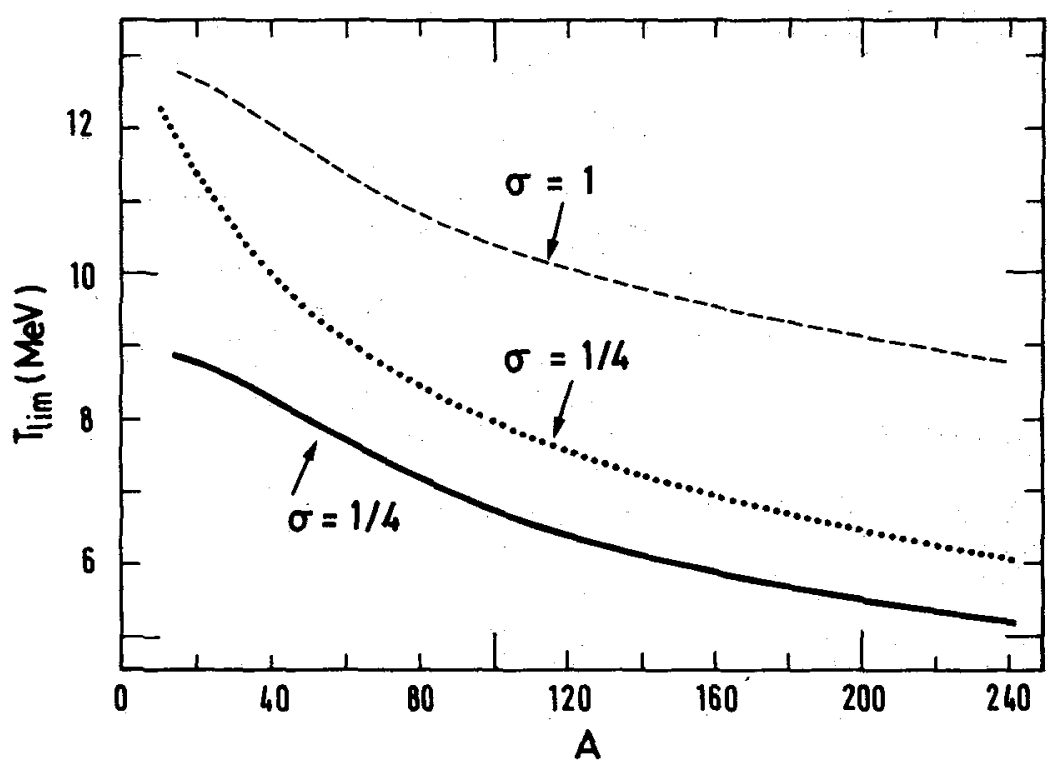

Fig. 1 - Limiting temperature obtained in the hot liquid drop model as a function of the nucleus mass number $/ 8 \%$. The results obtained for different effective interactions (see ref. /8/) are compared. High values of $\sigma$ are associated with high incompressibilities in nuclear matter.

for interaction SIII and for various temperatures

\begin{tabular}{|c|c|c|c|}
\hline$T(\mathrm{MeV})$ & 1 & 2 & 5 \\
\hline$\tau(s)$ & $2 \times 10^{-18}$ & $9 \times 10^{-20}$ & $1.3 \times 10^{-22}$ \\
\hline
\end{tabular}

Table 1 - Lifetime of lead-208, calculated in ref. /3/ for interaction SIII, at various temperatures.

It may be noted that lifetimes become comparable to reaction times for temperatures of the order of $5 \mathrm{MeV}$. The values in table 1 are, however, presumably underestimated $/ 3 /$.

When the density of the vapor becomes appreciable compared to the nuclear density, the evolution of the hot nucleus can no more be inferred from a static calculation. Furthermore what happens for temperature greater than $T_{\ell}$ is obviously beyond the scope of static calculations. Clearly a dynamical approach is needed. 


\section{II - TIME DEPENDENT HARTREE-FOCK CALCULATIONS}

The evolution of hot nuclei has recently been investigated by several variants of the dynamical mean-field approach. Let us quote the constrained Hartree-Fock method $/ 9 /$, the time dependent Thomas-Fermi approximation $/ 10 /$, the Vlasov equation $111 /$, the Monte-Carlo Hartree-Fock method $/ 12 \%$, and the time dependent Hartree-Fock (TDHF) approximation /13/. We will now describe some results which were recently obtained by the last approach $/ 14 /$.

Let us first recali that, in TDHF, the density matrix is assumed, at each time $t$, to be of the form given by eq. (1). The TDHF evolution equations for the orbitals $\varphi_{i}(\vec{r})$ and for the quantities $\alpha_{i}$ arising in eq. (1) preserve the occupation numbers, the single particle entropy, and the Hartree-Fock energy.

The results described below were obtained by numerical integration of the TDHF equations on a $0.2 \mathrm{fm}$ lattice containing 100 points, with a time step $\Delta t=0.25 \times 10^{-2,3} \mathrm{~s}$. The zero range density dependent force of Jaquaman, Mekjian and Zamick (JMZ) /15/ was used because of its simplicity and also because its parameters were adjusted to reproduce the saturation properties of nuclear matter : binding energy, density, and compression modulus. However it overbinds light nuclei. For instance $E / A=-11 \mathrm{MeV}$ in calcium-40.

In fig. 2 we show the evolution of a hot calcium-40, whose initial temperature is $T=6 \mathrm{MeV}$. The initial wave functions are taken to be pure oscillator functions, with a parameter $b=\sqrt{\hbar / \mathrm{m \omega}}=1.7 \mathrm{fm}$ adjusted to reproduce the root mean square (rms) radius of the Hartree-Fock ground state $(3.08 \mathrm{fm})$. The initial occupation numbers are determined from the Hartree-Fock single particle energies associated to the initial wave functions. It is assumed, in other words, that the residual interactions, during a first stage of the reaction, have thermalized the single particle degrees of freedom, and that the radius has not appreciably changed. Fig. 2 exhibits (see also fig. 6 below) the existence of a significant Landau damping of the monopole oscillation : the density distribution appears to reach a limit after two oscillations. This damping was already observed and discussed in zero temperature TDHF calculations $/ 16 /$. It arises because of the coupling to the continum states. It is this same coupling which leads to the emission of particles.

Figs. 3, 4 and 5 display the evolution of a hot calcium-40 for higher values of the initial temperature, namely $T=10,12$ and $15 \mathrm{MeV}$. Here also, the initial radius has been kept approximately equal to the Hartree-Fock ground state rms. radius. It can be seen on figs. $3-5$ that the amplitude of the monopole oscillation increases rapidly with temperature. A total vaporization of the nucleus occurs between 12 and $15 \mathrm{MeV}$; it will be discussed further in connection with the evolution of occupation numbers (table 2).

It is interesting to compare this result with that of the static calculations of sect. I. For the zero range interaction we are using and for the case of calcium-40 the limiting temperature $T_{\ell}$ discussed in sect. I is near $12 \mathrm{MeV}$. This value is in good agreement with the results of figures 4 and 5 .

In calculations using the BKN force (and the Vlasov equation) a higher value of the vaporization temperature was found (about $18 \mathrm{MeV}$ ) $/ 11$. This difference should probably be attributed to the $B K N$ force. Indeed this force leads to a large value of the compression modulus in nuclear matter; and this value is expected to produce a large value of the limiting temperature $T_{\ell}$ (see fig. 1 ).

In fig. 6 we show the evolution of the rms radius of the "residual nucleus" for the initial conditions $T=6,10$ and $15 \mathrm{MeV}$ and an initial calcium-40 nucleus. The residual nucleus is defined by truncating the single particle wave functions $\varphi_{i}(r)$ in eq. (1) at a cutoff radius $c=8 \mathrm{fm}$. This cutoff radius $c$ is larger than the nuclear radius, but much smaller than the size of the lattice used in the numerical solution $R=20 \mathrm{fm}$. The radius $c$ defines a separation between the residual nucleus (or liquid phase) and the emitted particles (or vapor phase). One may also observe on fig. 6 the increase with the temperature of the period 


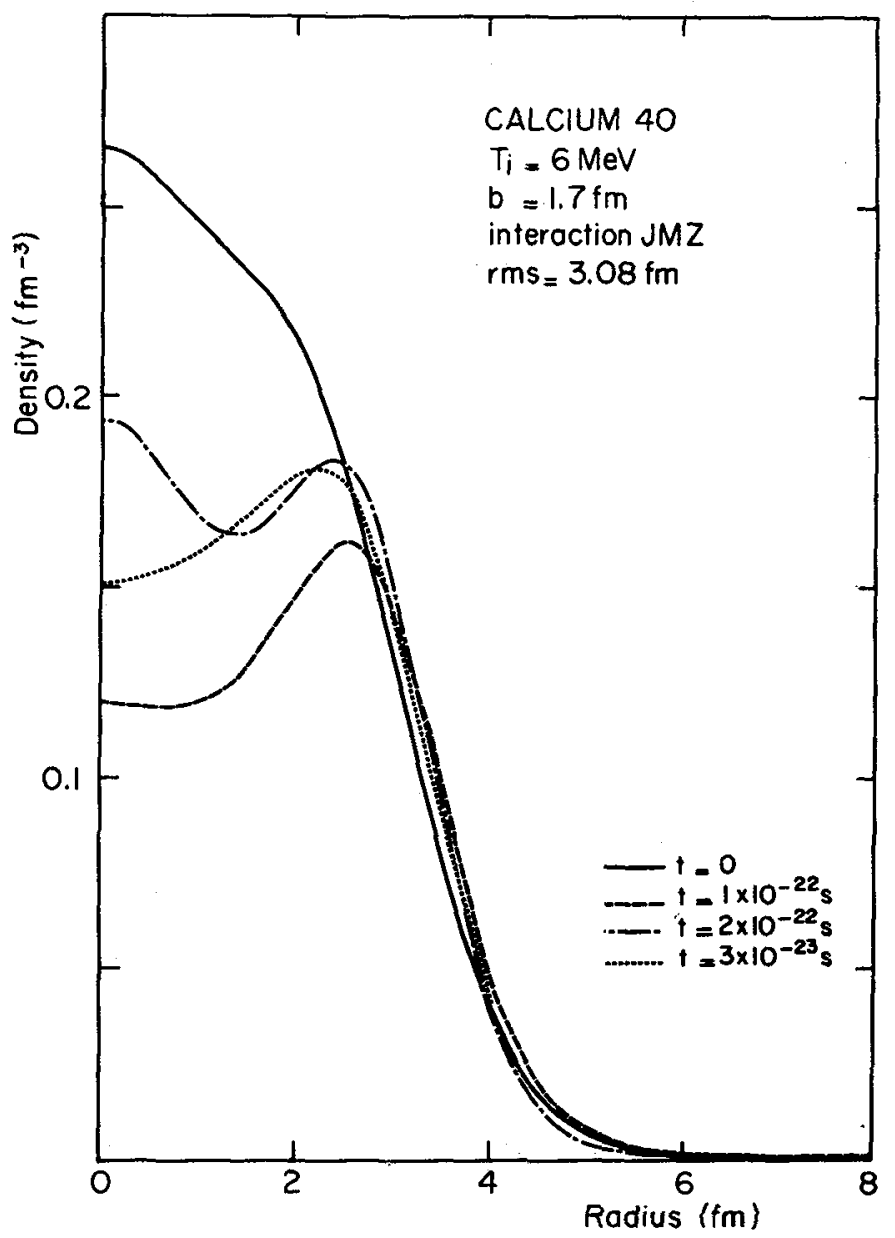

Fig. 2 - Time evolution of the density distribution for an initial nucleus $A=40$, $T=6 \mathrm{MeV}, b=1.7 \mathrm{fm}$. 


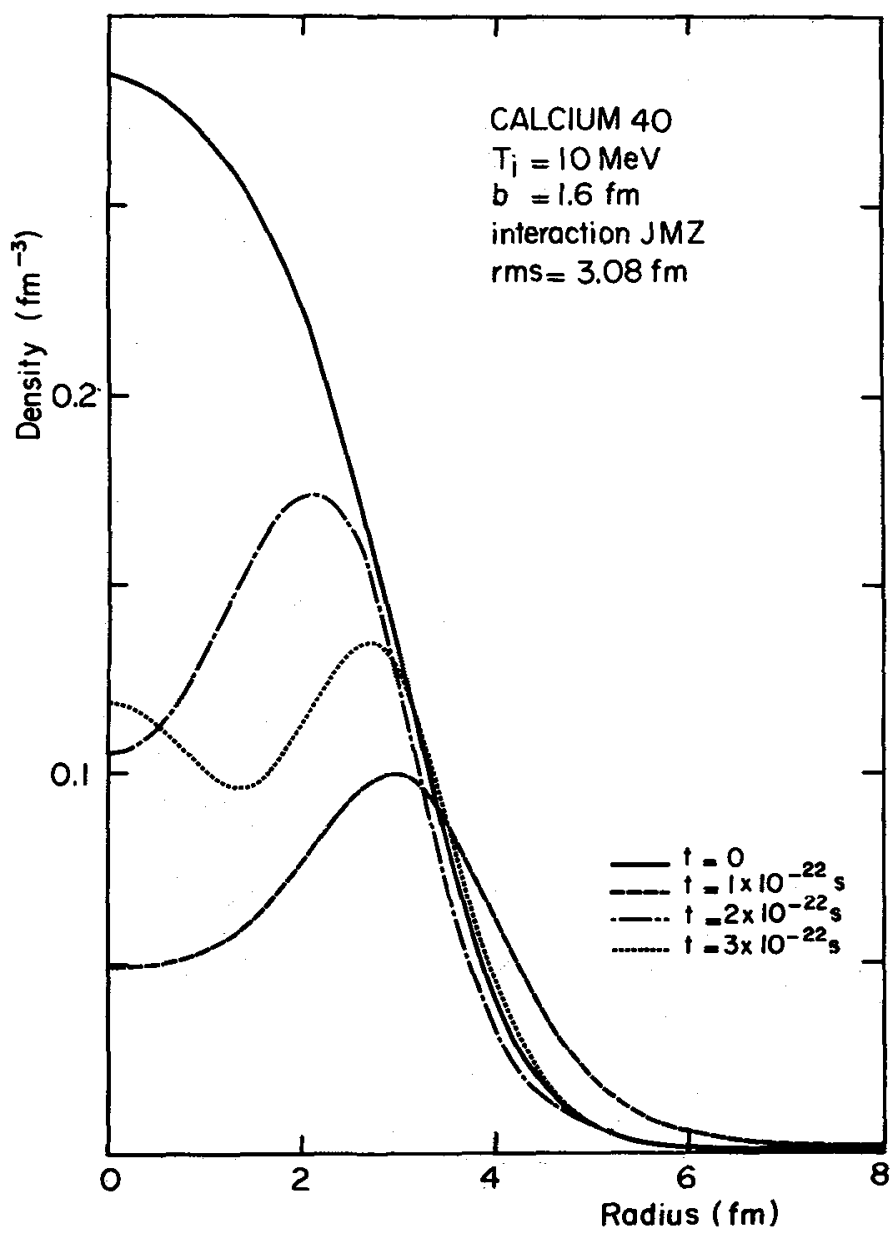

Fig. 3 - Time evolution of the density distribution for an initial nucleus $A=40$, $T=10 \mathrm{MeV}, \mathrm{b}=1.6 \mathrm{fm}$. 


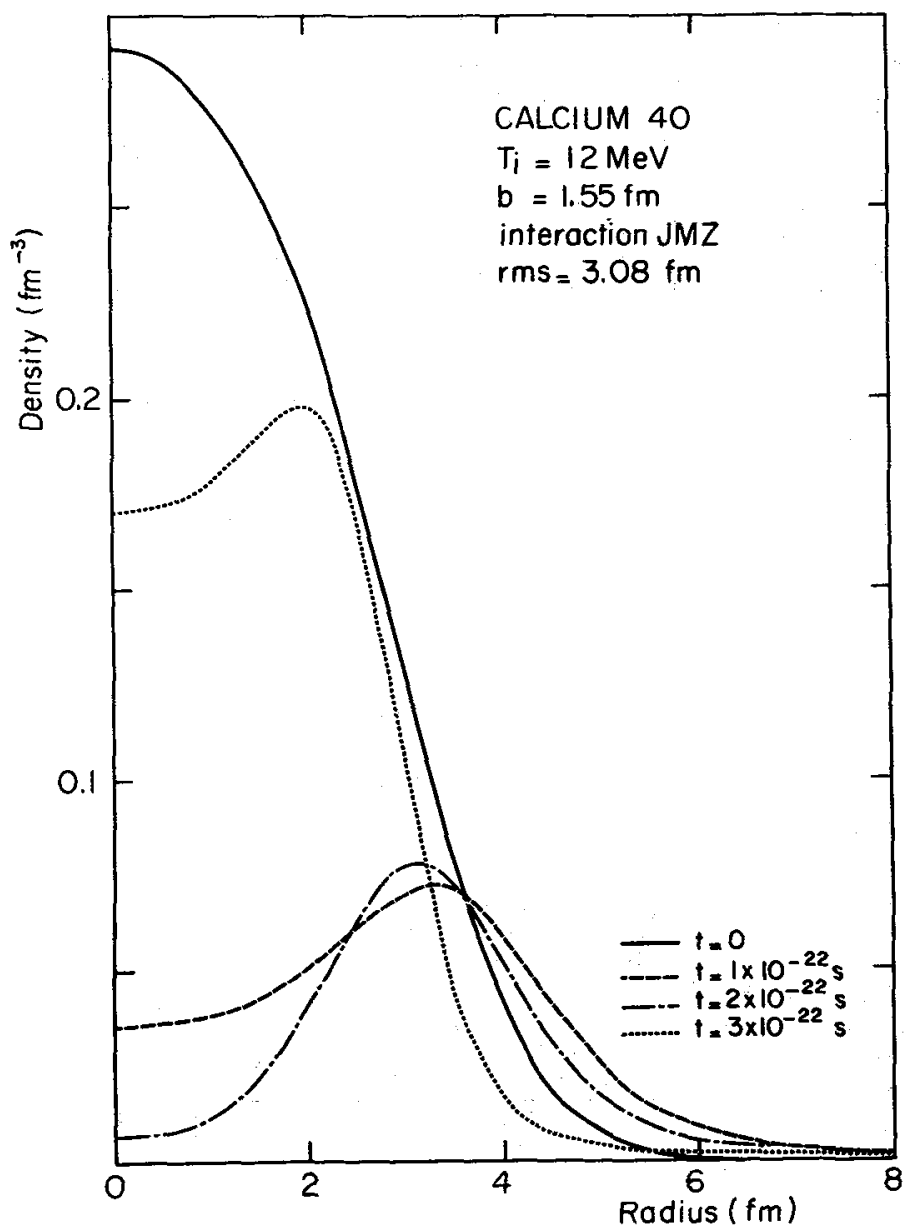

Fig. 4 - Time evolution of the density distribution for an initial nucleus $A=40$, $T=12 \mathrm{MeV}, b=1.55 \mathrm{fm}$. 


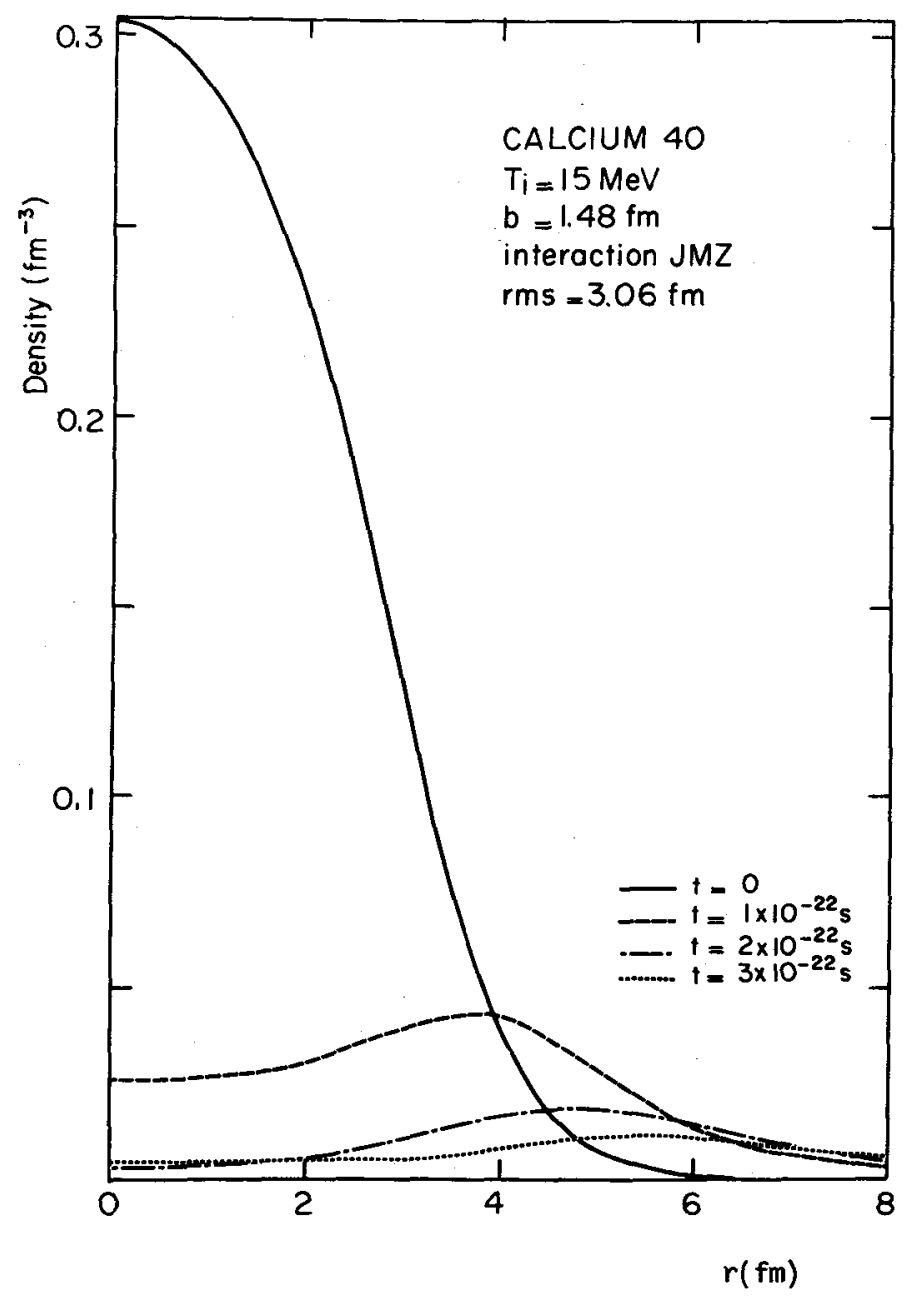

Fig. 5 - Time evolution of the density distribution for an initial nucleus $A=40$, $T=15 \mathrm{MeV}, b=1.48 \mathrm{fm}$. 


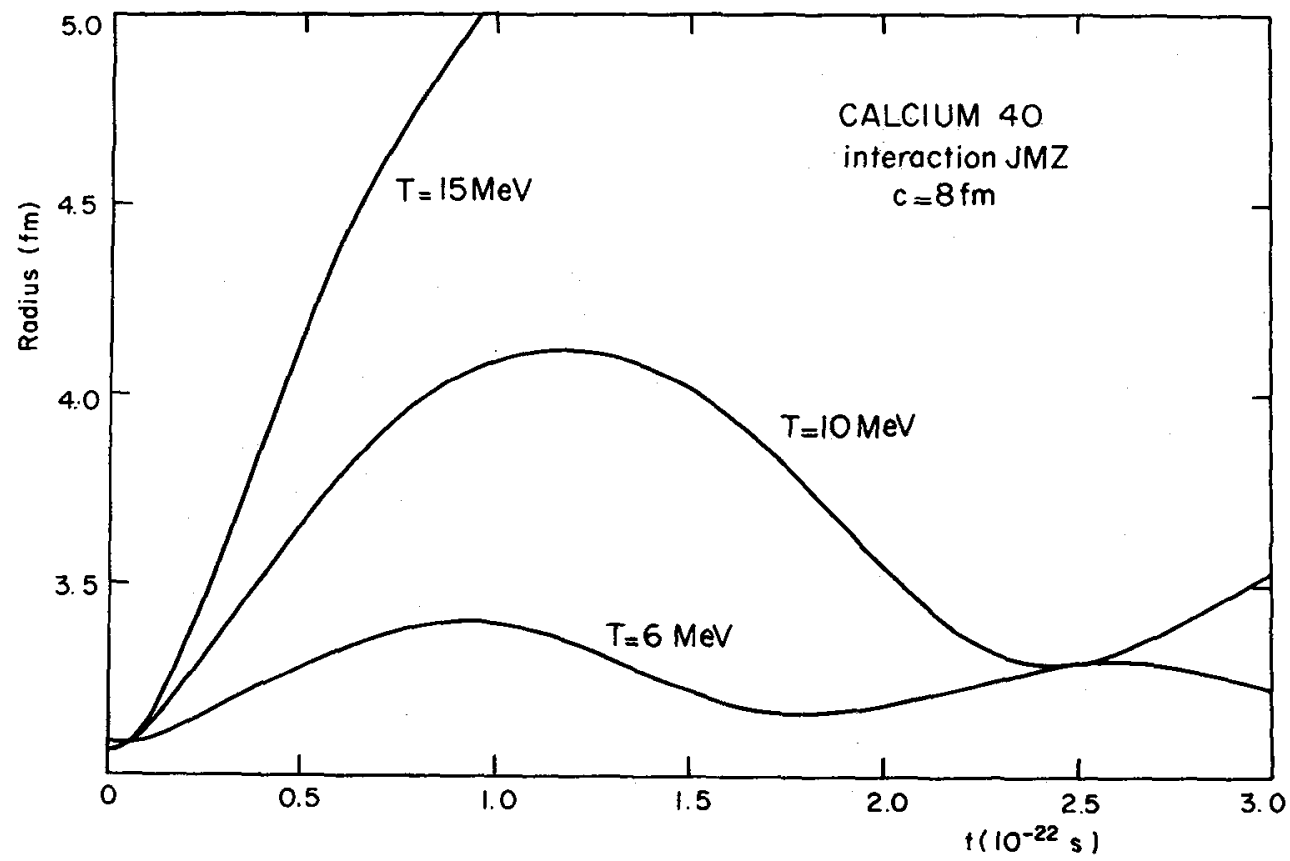

Fig. 6 - Time evolution of the root mean square radius of the residual nucleus for the initial conditions $A=40, T=6 \mathrm{MeV}, \mathrm{b}=1.7 \mathrm{fm} ; T=10 \mathrm{MeV}, \mathrm{b}=1.6 \mathrm{fm}$; $T=15 \mathrm{MeV}, \mathrm{b}=1.48 \mathrm{fm}$.

$\tau$ of the monopole oscillation. The corresponding energy $\hbar \times 2 \pi / \tau$ is $23.7 \mathrm{MeV}$ for $T=6 \mathrm{MeV}$ and $17.3 \mathrm{MeV}$ for $\mathrm{T}=10 \mathrm{MeV}$. For $T=15 \mathrm{MeV}$ the radius shows a monotonic increase with time, as can be expected from fig. 5 .

The particle emission leads of course to a decrease in time of the mass number and also of the energy per nucleon (i.e. to a cooling) of the residual nucleus as is $i l l$ ustrated by figs. 6 and 7 . (Recall that the total mass and the total Hartree-Fock energy are constants of the motion in TDHF). For initial temperatures up to $12 \mathrm{MeV}$ the average mass of the residual nucleus appears to reach a limit with time. The same remark holds for the energy per nucleon which goes asymptotically to a negative value. In contrast, for an initial temperature $T=15 \mathrm{MeV}$ the residual nucleus eventually disappears, while the energy per nucleon remains always positive. From figure 7 one can define schematically a lifetime $\tau$ of the hot nucleus as the time when the number of emitted particles is half its asymptotic value. For $T=6 \mathrm{MeV}$ this gives $\tau \simeq 1 \times 10^{-22} \mathrm{~s}$ in agreement with the value quoted in table 2. For higher temperatures such a definition of $T(T)$ is no longer meaningful since there is an important cooling ( $i . e$. decrease in E/A) over a time scale of $10^{-22} \mathrm{~s}$. The importance of this cooling in a short lapse of time suggests that the system may not have the opportunity to reach an initial condition with temperatures greater than some value around $10 \mathrm{MeV}$.

To gain a better understanding of the results shown in figs. 6 and 7 , it is useful to identify the origin of the emitted particles. To this end, we show in table 2 the occupation numbers $n_{i}$ at the initial time $t_{0}$ and, in the residual 


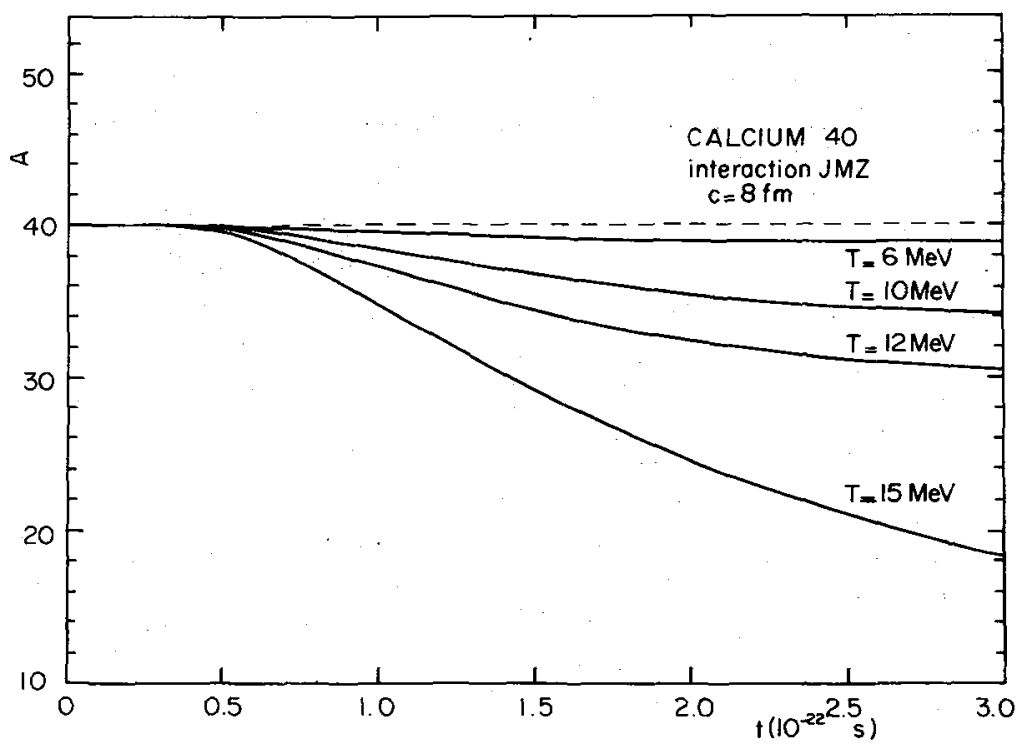

Fig. 7 - Time evolution of the average mass of the residual nucleus for various initial temperatures. The initial nucleus is calcium- 40 and the initial radius $3.08 \mathrm{fm}$.

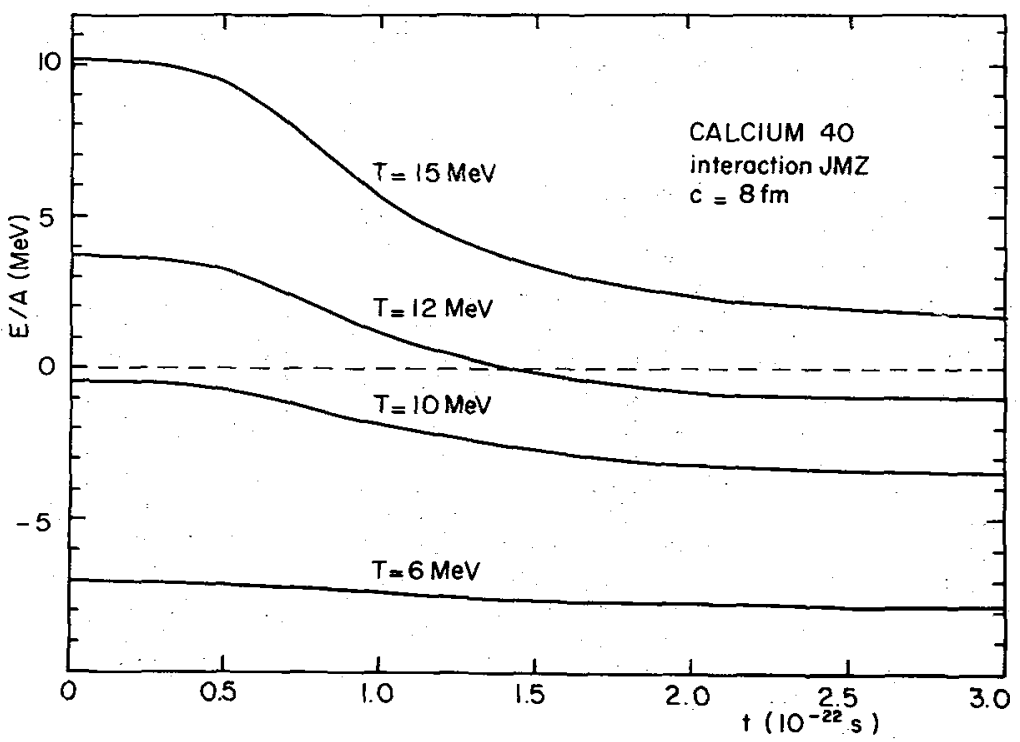

Fig. 8- Time evolution of the energy per nucleon in the residual nucleus for various initial temperatures. The initial nucleus is calcium-40 and the initial radius $3.08 \mathrm{fm}$. 
nucleus, at time $t_{1}=t_{0}+3 \times 10^{-22} \mathrm{~s}$. The ir calculation requires some care because the restrictions of the orbitals $\varphi_{i}(\vec{r})$ to the sphere of radius $c$ are no longer orthogona?. It is thus necessary to construct and diagonalize the restriction of the single particle density matrix in this sphere. In contrast with the global occupation numbers (which are constants of the motion) table 2 shows that the occupation numbers in the residual nucleus are modified during the TDHF evolution by the particle emission. It is worthwhile noting that the main effect of the particle emission is to empty unbound orbits in the initial Hartree-Fock potential. For $T=12 \mathrm{MeV}$ the entropy per nucleon decreases from $\mathrm{S} / \mathrm{A}=2.09$ to $\mathrm{S} / \mathrm{A}=1.51$ because the evaporated particles are the most disordered. Notice that for $\mathrm{T}$ greater than $10 \mathrm{MeV}$, the emitted particles produce a drastic reduction of the mean field depth ; at $T=15 \mathrm{MeV}$ this non-linear effect is sufficient to drive all the particles into the continuum, leading to total vaporization.

\begin{tabular}{|c|c|c|c|}
\hline$i$ & $E_{i}$ & $n_{i}\left(t_{0}\right)$ & $n_{i}\left(t_{1}\right)$ \\
\hline $1 s$ & -36.34 & $.9842+.00$ & $.9783+00$ \\
$1 \mathrm{p}$ & -28.09 & $.9402+00$ & $.9318+00$ \\
$1 \mathrm{~d}$ & -16.84 & $.7068+00$ & $.7027+00$ \\
$2 \mathrm{~s}$ & -12.49 & $.5386+00$ & $.5262+00$ \\
$1 \mathrm{f}$ & -3.52 & $.2075+00$ & $.2044+00$ \\
$2 \mathrm{p}$ & 1.54 & $.1011+00$ & $.7386-01$ \\
$\mathrm{Ig}$ & 10.70 & $.2386-01$ & $.1605-01$ \\
$2 \mathrm{~d}$ & 13.76 & $.1448-01$ & $.3587-03$ \\
$3 \mathrm{~s}$ & 14.38 & $.1308-01$ & $.7883-04$ \\
$\mathrm{lh}$ & 24.81 & $.2325-01$ & $.8123-04$ \\
\hline
\end{tabular}

Table 2 - Occupation numbers $n_{j}$ of the lowest orbitals at the initial time $t_{0}$, and at $t_{1}=t_{0}+3 \times 10^{-22} \mathrm{~s}$ in the residual nucleus. The initial condition is $A=40, \bar{T}=6 \mathrm{MeV}$, rms $=3.08 \mathrm{fm}$. The Hartree-Fock single particle energies $E_{i}(\mathrm{MeV})$ at time $t_{0}$ are also indicated.

\section{CONCLUSION AND OUTLOOK}

The time dependent Hartree-Fock formalism provides, through particle emission, a consistent dynamical treatment of the liquid and vapor nuclear phases. While being still numerically simple, it allows a comparison with the results of earlier static calculations, whose conclusions are in the overall confirmed.

of course important questions do remain. Some of our initial conditions are presumably unrealistic, because particle emission (and fragmentation) occuring during the first stage of the collision would prevent the nucleus to reach some of the assumed initial states. The effects of the residual interactions, which are expected to populate continuousiy the unbound orbitals, should be taken into account. 
Some aspects of nuclear fragmentation have already been investigated by different techniques $/ 12,17-19 /$. In the TDHF framework, an approximate way to study this question is through the random phase approximation (RPA) at finite temperature $/ 13,20 /$. In this approach and for a density-dependent interaction such as the JMZ force $115 /$, the effective "particle-hole" interaction was found to be repulsive at densities higher than the normal nuclear density and attractive at lower densities. This effect produces imaginary RPA roots which may reveal the onset of fragmentation at low nuclear density.

Let us finally recall that TDHF is only one among possible mean-field theories. Using a time dependent variational principle, it has been shown to be the "best" mean-field approximation for predicting the average values of singleparticle observables. When the quantities of interest, such as fluctuations or transition probabilities, are no more of the single-particle type, more sophisticated mean-field theories (which are suited to the evaluation of these quantities and which incorporate some correlation effects) may be derived from this same variational principle $/ 21 /$. In the zero temperature case, the mass dispersion of the residual nuclei has been evaluated by this method and the corrections /22-24/ found to improve significantly the "naive". TOHF evaluations which give too small values,

We hope that investigations, now in progress along these lines, will help to a better understanding of these exciting nuclear blobs, which our experimentalist friends excite, not far from here, with a tonic excitement.

\section{REFERENCES}

/1/ Küpper, W.A., Wegmann, G. and Hitf, E.R., Ann. Phys. (N.Y.) 88 (1974) 454.

12/ Sauer, G., Chandra, H. and Mose 1, U., Nucl. Phys. A264 (1976) 221 ,

13/ Bonche, P., Levit, S. and Vautherin, D., Nucl. Phys. 4427 (1984) 278 ; A436 (1985) 265.

14/ Strumberger, E., Bartel, J., Blin, A., Hiller, B. and Brack, M., Contribution to the present volume, p. 66 .

15/ Landau, L.D. and Lifschitz, E., Statistical Mechanics, Mir, Moscow, 1967, sect. 77.

16/ Tubbs, D.L. and Koonin, S.E., Ap. J. 232 (1979) L59.

17/ Suraud, E., ISN preprint, 1986.

18/ Levit, S. and Bonche, P., Nucl. Phys. A437 (1985) 426.

19/ Sagawa, H. and Bertsch, G.F., Phys. Lett. 155B (1985) 11.

/10/ Pi, M., Barranco, M., Nemeth, J., Ngô, C. and Tomasi, E., Phys. Lett. 166B (1986) 1.

111/ Vinet, L., Sebille, F., Gregoire, C. and Schuck, P., Preprint GANIL P 86-01.

/12/ Strack, B. and Knoll, J., Z. Phys. A315 (1984) 249.

113 / Vautherin, D. and Veneroni, M., Journat de Physique, in press.

114/ Vautherin, D. and Veneroni, M., preprint IPNO/TH 86-39.

/15/ Jaquaman, H., Mekjian, A.Z. and Zamick, L., Phys. Rev. C27 (1983) 2782.

$116 /$ Stringari, $S$, and Vautherin, D., Phys. Lett. 88B (1979) 1.

117/ Cugnon, J., Phys. Lett. 135B (1984) 374.

118/ Schulz, H., Kämpfer, B., Barz, H.W., Röpke, G. and Bondorf, J., Phys. Lett. $147 \mathrm{~B}$ (1984) 17.

/19/ Vicentini, A., Jacucci, G. and Pandharipande, V.R., Phys. Rev. C31 (1985) 1783.

120/ Vautherin, D. and Vinh Mau, N., Nucl. Phys. A422 (1984) 140.

/21/ Balian, R. and Veneroni, M., Ann. Phys. (N.Y.) 164 (1985) 334.

122/ Troudet, T. and Vautherin, D., Phys. Rev. C31 (1985) 278.

123/ Marston, J.B. and Koonin, S.E., Phys. Rev. Lett. 54 (1985) 1139.

/24/ Bonche, P. and Flocard, H., Nucl. Phys. A437 (1985) 189. 